\title{
Theory of Conductivity in Semiconducting Single-Wall Carbon Nanotubes
}

\author{
Shigeji Fujita ${ }^{1}$, Salvador Godoy ${ }^{2}$, Akira Suzuki ${ }^{3 *}$ \\ ${ }^{1}$ Department of Physics, University at Buffalo, Buffalo, USA \\ ${ }^{2}$ Departamento de Física, Facultad de Ciencias, Universidad Nacional Autónoma de México, Mexico City, Mexico \\ ${ }^{3}$ Department of Physics, Faculty of Science, Tokyo University of Science, Shinjuku-ku, Japan \\ Email: *asuzuki@rs.kagu.tus.ac.jp
}

Received August 13, 2012; revised September 16, 2012; accepted September 23, 2012

\begin{abstract}
The conduction of a single-wall carbon nanotube depends on the pitch. If there are an integral number of carbon hexagons per pitch, then the system is periodic along the tube axis and allows "holes" (not "electrons") to move inside the tube. This case accounts for a semiconducting behavior with the activation energy of the order of around $3 \mathrm{meV}$. There is a distribution of the activation energy since the pitch and the circumference can vary. Otherwise nanotubes show metallic behaviors (significantly higher conductivity). "Electrons" and "holes" can move in the graphene wall (two dimensions). The conduction in the wall is the same as in graphene if the finiteness of the circumference is disregarded. Cooper pairs formed by the phonon exchange attraction moving in the wall is shown to generate a temperature-independent conduction at low temperature $(3-20 \mathrm{~K})$.
\end{abstract}

Keywords: Semiconducting SWNT; Cartesian Unit Cell Model; Cooper Pair; Bloch Electron Dynamics

\section{Introduction}

Iijima [1] found after his electron diffraction analysis that carbon nanotubes ranging 4 to 30 nanometers $(\mathrm{nm})$ in diameter have helical multi-walled structures. Singlewall nanotube (SWNT) has about one nanometer in diameter and micrometers $(\mu \mathrm{m})$ in length. Ebbesen et al. [2] measured the electrical conductivity $\sigma$ of individual carbon nanotubes and found that $\sigma$ varies depending of the temperature $T$, the tube radius $r$ and the pitch $p$. Experiments show that SWNT can be either semiconducting or metallic, depending on how they are rolled up from the graphene sheets [3]. In the present work we shall present a microscopic theory of the electrical conductivity of semiconducting SWNT, starting with a graphene honeycomb lattice, developing a Bloch electron dynamics based on a rectangular cell model [4], and using kinetic theory. A SWNT can be formed by rolling a graphene sheet into a circular cylinder. The graphene which forms a honeycomb lattice is intrinsically anisotropic as we shall explain it in more detail later in Section 2. Moriyama et al. [5] fabricated 12 SWNT devices from one chip, and observed that two of the SWNT samples are semiconducting and the other 10 are metallic. The semiconducting SWNT samples show an activated-state tem-

${ }^{*}$ Corresponding author. perature behavior. That is, the resistance decreases with increasing temperature. Why are there two sets of samples showing very different behavior? The answer to this question is as follows.

The line passing the centers of the nearest-neighbor carbon hexagons forms a helical line around the nanotube with a pitch $p$ and a radius $r$.

In Figure 1(a), a section of the circular tube with a pitch $p$ is drawn. Its unrolled plane is shown in (b). The circumference $2 \pi r$ likely contains an integral number $m$ of the carbon hexagons (units). The pitch $p$, however, may or may not contain an integral number $n$ of units. In the fabrication process the pitch is not controlled. In the first alternative, the nanotube is periodic with the period $p$ along the tube axis. Then, there is a one-dimensional (1D) $k$-vector along the tube. A "hole" which has a positive charge $+e$ and a size of a unit ring of height $p$ and radius $r$ can go through inside the positively charged carbon wall. An "electron" having a negative charge $-e$ and a similar size is attracted by the carbon wall, and hence it cannot go straight inside the wall. Thus, there should be an extra "hole" channel current in a SWNT. Moriyama et al. [5] observed a "hole"-like current after examining the gate voltage effect. The system should have the lowest energy if the unit ring contains an integer set $(m, n)$ of carbon hexagons, which may be attained 


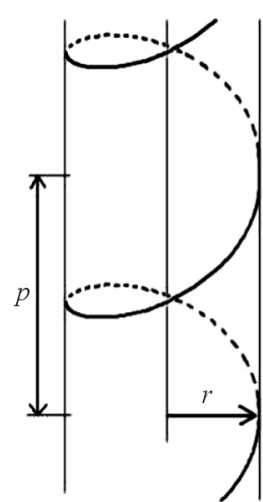

(a)

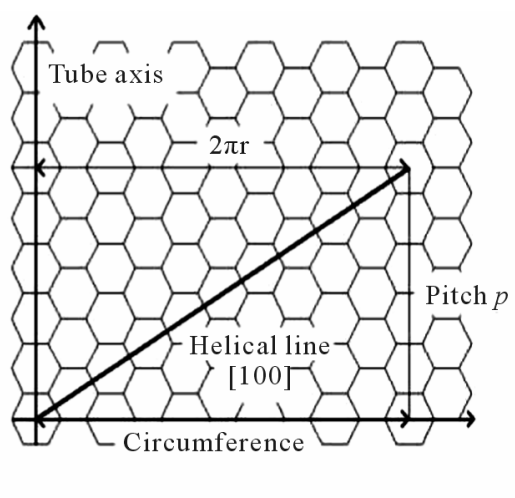

(b)
Figure 1. (a) A section circular tube wall with a radius $r$ and a pith $p$; (b) Its unrolled plane.

after annealing at high temperatures. This should happen if the tube length is comparable with the circumference. The experimental tube length is much greater than the circumference, and the pitch angle can be varied continuously. The set of irrational numbers is greater in cardinality than the set of rational numbers. Then, the first case in which the unit contains an integer set $(m, n)$ of hexagons must be the minority. This case then generates a semiconducting transport behavior. We shall show later that the transport requires an activation energy. Fujita and Suzuki [4] showed that the "electrons" and "holes" must be activated based on the rectangular unit cell model for graphene.

Saito, Dresselhaus and Dresselhaus [3] state that a SWNT is characterized by two integer indices $(m, n)$, for example, $m=n$ for an armchair nanotube whereas $m=0$ for a zigzag nanotube. If $n-m$ is a multiple of 3 , then a SWNT is metallic. They then argued that approximately one third of the SWNT are metallic, and the other two thirds are semiconducting. This model is in variance with the experimental observation by Moriyama et al. [5], where the majority of SWNT is metallic. We must look for a different classification scheme.

If a SWNT contains an irrational number of carbon hexagons, which happens more often, then the system does not allow a conduction along the tube axis. The system is still conductive since the conduction electrons ("electrons", "holes") can go through in the tube wall. This conduction is two-dimensional (2D), opposed to 1D, as can be seen in the unrolled configuration, which is precisely the graphene honeycomb lattice. This means that the conduction in the carbon wall should be the same as the conduction in graphene if the effect of the finiteness of the radius is neglected.

We consider graphene in Section 2. The current band theory of the honeycomb crystal is based on the WignerSeitz (WS) cell model $[3,6]$. The model applied to graphene predict a gapless semiconductor, which is not ob- served. The WS model [6] is suitable for the study of the ground-state energy of the crystal. To describe the Bloch electron dynamics [7] a new theory based on the Cartesian unit cell not matching with the natural triangular crystal axes must be used. Also the phonons can be discussed using Cartesian coordinate-systems, not with the triangular coordinate-systems. The conduction electron moves as a wave packet formed by the Bloch waves as pointed out by Ashcroft and Mermin in their book [7]. This picture is fully incorporated in our new theoretical model.

\section{Graphene}

We consider a graphene, in which carbon ions $\left(\mathrm{C}^{+}\right)$occupy a $2 \mathrm{D}$ honeycomb lattice, See Figure 2 . The normal current carriers are "electrons" and "holes". Following Ashcroft and Mermin [7], we adopt the semiclassical model of electron dynamics in solids. "Electrons" ("holes") are defined as quasi-electrons which move counterclockwise (clockwise) viewed from the tip of the applied magnetic field. In the semiclassical (wave packet) theory, it is necessary to introduce a $\boldsymbol{k}$-vector:

$$
\boldsymbol{k}=\boldsymbol{k}_{x} \hat{\boldsymbol{e}}_{x}+\boldsymbol{k}_{y} \hat{\boldsymbol{e}}_{y}+\boldsymbol{k}_{z} \hat{\boldsymbol{e}}_{z},
$$

where $\hat{\boldsymbol{e}}_{x}, \hat{\boldsymbol{e}}_{y}, \hat{\boldsymbol{e}}_{z}$ are the Cartesian orthonormal vectors, since the $\boldsymbol{k}$-vectors are involved in the semiclassical equation of motion:

$$
\hbar \dot{\boldsymbol{k}} \equiv \hbar \frac{\mathrm{d} \boldsymbol{k}}{\mathrm{d} t}=q(\boldsymbol{E}+\boldsymbol{v} \times \boldsymbol{B}),
$$

where $q$ is the charge of a conduction electron, and $\boldsymbol{E}$ and $\boldsymbol{B}$ are the electric and magnetic fields, respectively. The vector

$$
\boldsymbol{v} \equiv \frac{1}{\hbar} \frac{\partial \varepsilon}{\partial \boldsymbol{k}}
$$

is the electron velocity, where $\varepsilon=\varepsilon(\boldsymbol{k})$ is the energy. The $2 \mathrm{D}$ crystals such as graphene can also be treated similarly, only the $Z$-component being dropped. The choice of the Cartesian axes and the unit cell is obvious for the cubic crystals. We must choose an orthogonal unit cell also for the honeycomb lattice, as demonstrated below.

Graphene forms a 2D honeycomb lattice. The WS unit cell is a rhombus shown in Figure 2(a).

The potential energy $V(\boldsymbol{r})$ is lattice-periodic:

$$
V\left(\boldsymbol{r}+\boldsymbol{R}_{m n}\right)=V(\boldsymbol{r}),
$$

where

$$
\boldsymbol{R}_{m n} \equiv m \boldsymbol{a}_{1}+n \boldsymbol{a}_{2},
$$

is a Bravais vector with the primitive vectors $\left(\boldsymbol{a}_{1}, \boldsymbol{a}_{2}\right)$ and integers $(m, n)$. In the field theoretical formulation the field point $\boldsymbol{r}$ is given by 


$$
\boldsymbol{r}=\boldsymbol{r}^{\prime}+\boldsymbol{R}_{m n}
$$

where $\boldsymbol{r}^{\prime}$ is the point defined within the standard unit cell. Equation (4) describes the 2D lattice periodicity but does not establish the $\boldsymbol{k}$-space, which is explained below.

To see this clearly, we first consider an electron in a simple square (sq) lattice. The Schrödingier wave equation is

$$
\mathrm{i} \hbar \frac{\partial}{\partial t} \psi(\boldsymbol{r})=-\frac{\hbar^{2}}{2 m^{*}} \nabla^{2} \psi(\boldsymbol{r})+V_{\mathrm{sq}}(\boldsymbol{r}) \psi(\boldsymbol{r}) .
$$

The Bravais vector for the sq lattice $\boldsymbol{R}_{m n}^{(0)}$ is

$$
\begin{gathered}
\boldsymbol{R}_{m n}^{(0)} \equiv m \boldsymbol{a}_{x}+n \boldsymbol{a}_{y}=m a \hat{\boldsymbol{e}}_{x}+n a \hat{\boldsymbol{e}}_{y}, \\
(a=\text { lattice constant })
\end{gathered}
$$

The system is lattice periodic:

$$
V_{\mathrm{sq}}\left(\boldsymbol{r}+\boldsymbol{R}_{m n}^{(0)}\right)=V_{\mathrm{sq}}(\boldsymbol{r}) .
$$

If we choose a set of Cartesian coordinates $(x, y)$ along the sq lattice, then the Laplacian term in Equation (7) is given by

$$
\nabla^{2} \psi(x, y)=\left(\frac{\partial^{2}}{\partial x^{2}}+\frac{\partial^{2}}{\partial y^{2}}\right) \psi(x, y) .
$$

If we choose a periodic square boundary with the side length $\mathrm{Na}, N=$ integer, then there are 2D Fourier transforms and $\boldsymbol{k}$-vectors.

We now go back to the original graphene system. If we choose the $x$-axis along either $\boldsymbol{a}_{1}$ or $\boldsymbol{a}_{2}$, then the potential energy field $V(\boldsymbol{r})$ is periodic in the $x$-direction but it is aperiodic in the $y$-direction. For an infinite lattice the periodic boundary is the only acceptable boundary condition for the Fourier transformation. Then, there is no 2D $\boldsymbol{k}$-space spanned by 2D $\boldsymbol{k}$-vectors. If we omit the kinetic energy term, then we can still use Equation (4) and obtain the ground state energy (except the zero point energy).

We now choose the orthogonal unit cell shown in Figure 2(b). The unit has side lengths

$$
b_{1}=\sqrt{3} a_{0}, b_{2}=3 a_{0},
$$

where $a_{0}$ is the nearest neighbor distance between two C's. The unit cell has four (4) C's. The system is latticeperiodic in the $x$ - and $y$-directions, and hence there are $2 \mathrm{D} k$-space.

The "electron" ("hole") is defined as a quasi-electron which has an energy higher (lower) than the Fermi energy $\varepsilon_{F}$ and "electron" ("hole") are excited on the positive (negative) side of the Fermi surface with the convention that the positive normal vector at the surface points in the energy-increasing direction.

The "electron" (wave packet) may move up or down along the $y$-axis to the neighboring unit cells passing

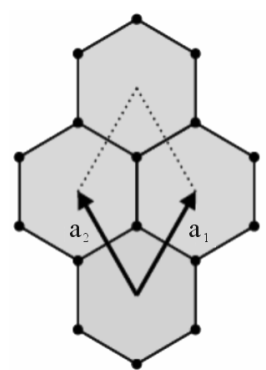

(a)

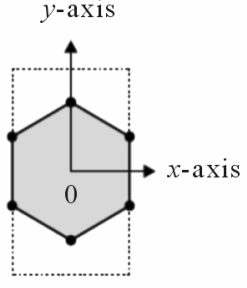

(b)
Figure 2. (a) WS unit cell, rhombus (dotted lines) for graphene; (b) The orthogonal unit cell, rectangle (dotted lines).

over one $\mathrm{C}^{+}$. The positively charged $\mathrm{C}^{+}$acts as a welcoming (favorable) potential valley for the negatively charged "electron", while the same $\mathrm{C}^{+}$act as a hindering potential hill for the positively charged "hole". The "hole", however, can move horizontally along the $x$-axis without meeting the hindering potential hills. Thus the easy channel directions for the "elections" ("holes") are along the $y$ - $(x-)$ axes.

Let us consider the system (graphene) at $0 \mathrm{~K}$. If we put an electron in the crystal, then the electron should occupy the center $\mathrm{O}$ of the Brillouin zone, where the lowest energy lies. Additional electrons occupy points neighboring the center $\mathrm{O}$ in consideration of Pauli's exclusion principle. The electron distribution is lattice-periodic over the entire crystal in accordance with the Bloch theorem [8].

Carbon (C) is a quadrivalent metal. The first few lowlying energy bands are completely filled. The uppermost partially filled bands are important for transport properties discussion. We consider such a band. The (2D) Fermi surface, which defines the boundary between the filled and unfilled $\boldsymbol{k}$-space (area) is not a circle since the $x-y$ symmetry is broken $(b \neq c)$. The "electron" effective mass is lighter in the direction [110] than perpendicular to it. Hence the electron motion is intrinsically anisotropic. The negatively charged "electron" is near the positive ions $\mathrm{C}^{+}$. Hence, the gain in the Colulomb interaction is greater for "electron". Thus, the "electrons" are the majority carriers at zero gate voltage. That is, the system has two different masses and it is intrinsically anisotropic. If the electron number is raised by the gate voltage applied perpendicular to the plane, then the Fermi surface more quickly grows in the easy-axis $(y$-) direction than in the $x$-direction. The Fermi surface must approach the Brillouin boundary at right angles because of the inversion symmetry possessed by honeycomb lattice [9]. Then at a certain voltage, a "neck" Fermi surface must be developed.

The same easy channels in which the "electron" runs with a small mass, may be assumed for other hexagonal directions, [011] and [101]. The currents run in the three channels $\langle 110\rangle \equiv[110],[011]$, and $[101]$. The total 
current (magnitude) along the field direction $\mu$ is proportional to [10]

$$
\begin{aligned}
& \sum_{\kappa \text { channels }} \cos ^{2}(\mu, \kappa) \\
= & \cos ^{2} \theta+\cos ^{2}(\theta+2 \pi / 3)+\cos ^{2}(\theta-2 \pi / 3)=3 / 2 .
\end{aligned}
$$

Note that this current does not depend on the angle $\theta$ between the field direction $(\mu)$ and the channel current direction $(\kappa)$. More detailed discussion can be found in ref. [10]. Hence, the graphene does not show anisotropy in the conductivity.

We have seen earlier that the "electron" and "hole" have different internal charge distributions and therefore have different effective masses. Which carriers are easier to be activated or excited? The "electron" is near the positive ions and the "hole" is farther away from the ions. Hence, the gain in the Coulomb interaction is greater for the "electron". That is, the "electrons" are more easily activated (or excited). The presence of the welcoming $\mathrm{C}^{+}$ ions in the channel direction also enhances this inequality. [6]

We may represent the activation energy difference by

$$
\varepsilon_{1}<\varepsilon_{2}
$$

The thermally-activated (or excited) electron densities are given by

$$
n_{j}(T)=n_{j} \exp \left(-\varepsilon_{j} / k_{\mathrm{B}} T\right)
$$

where $j=1$ and 2 represent the "electron" and "hole", respectively. The prefactor $n_{j}$ is the density at hightemperature limit.

\section{SWNT}

Let us consider the long SWNT rolled with a graphene sheet. The charge may be transported by the channeling "electrons" and "holes" in the graphene wall. But the "holes" within the wall can also contribute to the charge transport. Because of this extra channel inside the carbon nanotube, "holes" are likely to be the majority carriers in nanotubes although "electrons" are the dominant carriers in graphene. Moriyama et al. [5] studied the electrical transport in semiconducting SWNT in the temperature range $2.6-200 \mathrm{~K}$, and found from the filed (gate voltage) effect that the carriers are "hole"-like. Their data are reproduced in Figure 3.

The conductivity depends on the pitch of the SWNT. The helical line is defined as the line passing the nearest neighbors of the $\mathrm{C}$-hexagons. The helical angle $\theta$ is the angle between the helical line and the tube axis. The degree of helicity $h$ may be defined as

$$
h \equiv \cos \theta .
$$

For a macroscopically large graphene the conductivity

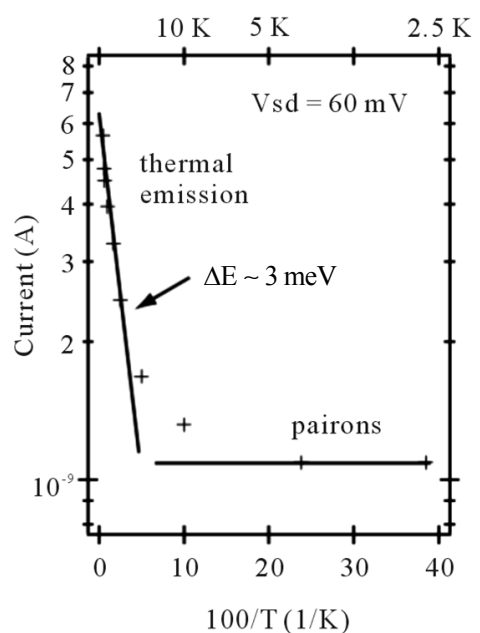

Figure 3. Log-scale plot of the currents in semiconducting SWNT as a function of inverse temperature after Moriyama et al. [5].

is isotropic as we saw in Section 2. The conductivity $\sigma$ in (semiconducting) SWNT depends on this helicity $h$. This is a kind of a finite size effect. The circumference is finite while the tube length is macroscopic.

In a four-valance-electron conductor such as graphene all electrons are bound to ions at $0 \mathrm{~K}$, and there is no conduction. If a "hole" having the charge $+e$ and the size of a unit ring is excited, then this "hole" can move along the tube axis with the activation energy $\varepsilon_{3}$ and the effective mass $m_{3}$. Both $\varepsilon_{3}$ and $m_{3}$ depend on the radius and the pitch.

We are now ready to discuss the conductivity of semiconducting SWNT. There are four currents carried by

1) "Electrons" moving in the graphene wall with the mass $m_{1}$ and the density $n_{1} \exp \left(-\varepsilon_{1} / k_{\mathrm{B}} T\right)$, running in the channels $\langle 110\rangle$.

2) "Holes" moving in the graphene wall with the mass $m_{2}$ and the density $n_{2} \exp \left(-\varepsilon_{2} / k_{\mathrm{B}} T\right)$, running in the channels $\langle 100\rangle$.

3) "Holes" moving with the mass $m_{3}$ and the density $n_{3} \exp \left(-\varepsilon_{3} / k_{\mathrm{B}} T\right)$, running in the tube-axis direction. The activation (or excitation) energy $\varepsilon_{3}$ and the effective mass $m_{3}$ vary with the radius and the pitch.

4) Cooper pairs (pairons) formed by the phononexchange attraction, which move in the graphene wall.

In actuality, one of the currents may be dominant, and be observed.

In the normal Ohmic conduction due to the conduction electrons the resistance is proportional to the sample (tube) length. Then, the conductivity $\sigma$ is given by the Drude formula:

$$
\sigma=\frac{n q^{2} \tau}{m^{*}} \equiv \frac{n q^{2}}{m^{*}} \frac{1}{\Gamma},
$$

where $q$ is the carrier charge $( \pm e), m^{*}$ the effective 
mass, $n$ the carrier density, and $\tau$ the relaxation (collision) time. The relaxation rate $\Gamma \equiv \tau^{-1}$ is the inverse of the relaxation time. If the impurities and phonons are scatterers, then the rate $\Gamma$ is the sum of the impurity scattering rate $\Gamma_{\text {imp }}$ and the phonon scattering rate $\Gamma_{\mathrm{ph}}(T)$ :

$$
\Gamma=\Gamma_{\text {imp }}+\Gamma_{\mathrm{ph}}(T) .
$$

The impurity scattering rate $\Gamma_{\text {imp }}$ is temperatureindependent and the phonon scattering rate $\Gamma_{\mathrm{ph}}(T)$ is temperature $(T)$-dependent. The phonon scattering rate $\Gamma_{\mathrm{ph}}(T)$ is linear in $T$ above around $2 \mathrm{~K}$ :

$$
\Gamma_{\mathrm{ph}}(T)=a T, a=\text { constant, (above } 2 \mathrm{~K} \text { ). }
$$

The temperature dependence should arise from the carrier density $n(T)$ and the phonon scattering rate $\Gamma_{\mathrm{ph}}(T)$. Writing the $T$-dependence explicitly, we obtain from Equations (14)-(18)

$$
\sigma=\sum_{j} \frac{e^{2}}{m_{j}^{*}} n_{j} \mathrm{e}^{-\varepsilon_{j} / k_{\mathrm{B}} T} \frac{1}{\Gamma_{\mathrm{imp}}+a T} .
$$

Moriyama et al. [5] used the Arrhenius plot for the data above $20 \mathrm{~K}$ and obtained the activation energy

$$
\varepsilon_{3} \sim 3 \mathrm{meV} \text {. }
$$

By studying the field (gate voltage) effect, the carriers were found to be "hole"-like. Thus, the major currents observed can be interpreted in terms of the "holes" moving within the tube wall.

This "hole" axial transport depends on the unit ring containing $m \times n$ hexagons. Since the pitch and the circumference have distributions, the activation energy $\varepsilon_{3}$ should also have a distribution. Hence the obtained value in Equation (20) must be regarded as the averaged value.

Liu et al. [10] systematically measured the resistance $\rho(T)$ of SWNT under hydrostatic pressures, and fitted their data by using 2D variable range hopping (vrh) theoretical formula [11]:

$$
\rho(T)=\rho_{0} \exp \left(T_{0} / T\right)^{1 / 3},
$$

where

$$
T_{0}=525 \mathrm{~K}
$$

is a fit (temperature) parameter and $\rho_{0}$ a (resistance) parameter. Mott's vrh theory [12] is applicable when highly random disorders exist in the system. An individual SWNT (annealed) is unlikely to have such randomness. We take a different view here. The scatterings are due to impurities and phonons. But carriers ("holes") have a distribution in the unit cell size. Hence the distribution of the activation energy introduces the flattening of the Arrhenius slope by the factor 1/3. Compare Equation (21) and Equation (14).

The "hole" size is much greater than the usually as- sumed atomic impurity size and the phonon size, which are of the order of the lattice constant. This size mismatch may account for a ballistic charge transport observed by Frank et al. [13] and others [14]. More careful studies are required to establish the cause of the ballisticity.

We now go back to the data shown in Figure 3. Below $20 \mathrm{~K}$ the currents observed are very small and they appear to approach a constant in the low temperature limit (large $T^{-1}$ limit). These currents, we believe, are due to the Cooper pairs.

The Cooper pairs (pairons) move in 2D with the linear dispersion relation [15]:

$$
\begin{aligned}
\varepsilon & =c^{(j)} p, \\
c^{(j)} & =(2 / \pi) v_{\mathrm{F}}^{(j)},
\end{aligned}
$$

where $v_{\mathrm{F}}^{(j)}$ is the Fermi velocity of the "electron" $(j=1)$ ["hole" $(j=2)$ ].

Consider first "electron"-pairs. The velocity $\boldsymbol{v}$ is given by (omitting superscript)

$$
\boldsymbol{v}=\frac{\partial \varepsilon}{\partial \boldsymbol{p}} \text { or } v_{x}=\frac{\partial \varepsilon}{\partial p} \frac{\partial p}{\partial p_{x}}=c \frac{p_{x}}{p},
$$

where

$$
p \equiv \sqrt{p_{x}^{2}+p_{y}^{2}}
$$

The equation of motion along the $\boldsymbol{E}$-field $(x-)$ direction is

$$
\frac{\partial p_{x}}{\partial t}=q^{\prime} E
$$

where $q^{\prime}$ is the charge $\pm 2 e$ of a pairon. The solution of Equation (27) is given by

$$
p_{x}=q^{\prime} E t+p_{x}^{(0)},
$$

where $p_{x}^{(0)}$ is the initial momentum component. The current density $j_{\mathrm{p}}$ is calculated from (charge $q^{\prime}$ ) $\times$ (number density $\left.n_{\mathrm{p}}\right) \times($ average velocity $\overline{\boldsymbol{v}}$ ). The average velocity $\overline{\boldsymbol{v}}$ is calculated by using Equations (25) and (28) with the assumption that the pair is accelerated only for the collision time $\tau$. We then obtain

$$
j_{\mathrm{p}} \equiv q^{\prime} n_{\mathrm{p}} \bar{v}=q^{\prime} n_{\mathrm{p}} c \frac{1}{p}\left(q^{\prime} E \tau\right)=q^{\prime 2} \frac{c}{p} n_{\mathrm{p}} E \tau .
$$

For stationary currents, the pairon density $n_{\mathrm{p}}$ is given by the Bose distribution function $f\left(\varepsilon_{p}\right)$ :

$$
n_{\mathrm{p}}=f\left(\varepsilon_{p}\right) \equiv\left[\exp \left(\varepsilon_{p} / k_{\mathrm{B}} T-\alpha\right)-1\right]^{-1},
$$

where $e^{\alpha}$ is the fugacity. Integrating the current $j_{\mathrm{p}}$ over all 2D $p$-space, and using Ohm's law $j=\sigma E$ we obtain for the conductivity $\sigma$ :

$$
\sigma=(2 \pi \hbar)^{-2} q^{\prime 2} c \int \mathrm{d}^{2} p p^{-1} f\left(\varepsilon_{p}\right) \tau .
$$


In the temperature ranging between 2 and $20 \mathrm{~K}$ we may assume the Boltzmann distribution function for $f\left(\varepsilon_{p}\right):$

$$
f\left(\varepsilon_{p}\right) \simeq \exp \left(\alpha-\varepsilon_{p} / k_{\mathrm{B}} T\right) .
$$

We assume that the relaxation time arises from the phonon scattering so that $\tau=(a T)^{-1}$, see Equations (16)-(18). After performing the $p$-integration we obtain

$$
\sigma=\frac{2}{\pi} \frac{e^{2} k_{\mathrm{B}}}{a \hbar^{2}} e^{\alpha},
$$

which is temperature-independent. If there are "electrons" and "hole" pairs, they contribute additively to the conductivity. These pairons should undergo a Bose-Einstein condensation at a temperature lower than $2.2 \mathrm{~K}$. We predict a superconducting state at lower temperatures.

\section{Summary and Discussion}

A SWNT is likely to have an integral number of carbon hexagons around the circumference. If each pitch contains an integral number of hexagons, then the system is periodic along the tube axis, and "holes" (and not "electrons") can move along the tube axis. The system is semiconducting with an activation energy $\varepsilon_{3}$. This energy $\varepsilon_{3}$ has a distribution since both pitch and circumference have distributions. The pitch angle is not controlled in the fabrication processes. There are more numerous cases where the pitch contains an irrational numbers of hexagons. In these cases the system shows a metallic behavior experimentally [16].

In the process of arriving at our main conclusion we have uncovered the following results.

1) "Electrons" and "holes" can move in $2 \mathrm{D}$ in the carbon wall in the same manner as in graphene.

2) For a metallic SWNT 1) implies that the conduction in the wall shows no pitch dependence.

3) The Cooper pairs are formed in the wall. They should undergo Bose-Einstein condensation at lower temperature, exhibiting a superconducting state.

A metallic SWNT will be treated separately.

\section{REFERENCES}

[1] S. Iijima, "Helical Microtubules of Graphitic Carbon," Nature, Vol. 354, 1991, pp. 56-58. doi:10.1038/354056a0

[2] T. W. Ebbesen, H. J. Lezec, H. Hiura, J. W. Bennet, H. F. Ghaemi and T. Thio, "Electrical Conductivity of Individual Carbon Nanotubes," Nature, Vol. 382, 1996, pp. 54-

\section{6. doi: $10.1038 / 382054 \mathrm{a} 0$}

[3] R. Saito, G. Dresselhaus and M. S. Dresselhaus, "Physical Properties of Carbon Nanotubes," Imperial College Press, London, 1998, pp. 35-58. doi:10.1142/9781860943799 0003

[4] S. Fujita and A. Suzuki, "Theory of Temperature Dependence of the Conductivity in Carbon Nanotubes," Journal of Applied Physics, Vol. 107, 2010, Article ID: 013711. doi:10.1063/1.3280035

[5] S. Moriyama, K. Toratani, D. Tsuya, M. Suzuki, Y. Aoyagi and K. Ishibashi, "Electrical Transport in Semiconducting Carbon Nanotubes," Physica E, Vol. 24, No. 1-2, 2004, pp. 46-49. doi:10.1016/i.physe.2004.04.022

[6] E. Wigner and F. Seitz, "On the Constitution of Metallic Sodium," Physical Review, Vol. 43, No. 10, 1933, pp. 804-810. doi:10.1103/PhysRev.43.804

[7] N. W. Ashcroft and N. D. Mermin, "Solid State Physics," Saunders, 1976, pp. 91-93, 217, 228, 229.

[8] F. Bloch, "Über die Quantenmechanik der Elektronen in Kristallgittern," Zeit Physik, Vol. 52, 1928, pp. 555-599.

[9] S. Fujita and K. Ito, "Quantum Theory of Conducting Matter," Springer, New York, 2007, pp. 85-90,106-107. doi:10.1007/978-0-387-74103-1

[10] S. Fujita, A. Garcia, D. O'Leyar, S. Watanabe and T. Burnett, "On the Conductance of a Lattice-Like Network," Journal of Physics and Chemistry of Solids, Vol. 50, No. 1, 1989, pp. 27-31. doi:10.1016/0022-3697(89)90468-X

[11] B. Liu, B. Sundqvist, O. Andersson, T. Wågberg, E. B. Nyeanchi, X.-M. Zhu and G. Zou, "Electric Resistance of Single-Walled Carbon Nanotubes under Hydrostatic Pressure," Solid State Communications, Vol. 118, No. 1, 2001, pp. 31-36. doi:10.1016/S0038-1098(01)00034-5

[12] N. F. Mott, "Conduction in Non-Crystalline Materials," Oxford University Press, Oxford, 1987.

[13] S. Frank, P. Poncharal, Z. I. Wang and W. A. de Heer, "Carbon Nanotube Quantum Resistors," Science, Vol. 280, No. 5370, 1998, pp. 1744-1746. doi:10.1126/science. 280.5370 .1744

[14] A. Javey, J. Guo, Q. Wang, M. Lundstrom and H. Dai, "Ballistic Carbon Nanotube Field-Effect Transistors," Nature, Vol. 424, 2003, pp. 654-657. doi:10.1038/nature01797

[15] S. Fujita, K. Ito and S. Godoy, "Quantum Theory of Conducting Matter: Superconductivity," Springer, New York, 2009, pp. 77-79.

[16] S. J. Tans, M. H. Devoret, H. Dai, A. Thess, R. E. Smalley, L. J. Geerligs and C. Dekker, "Individual Single-Wall Carbon Nanotubes as Quantum Wires," Nature, Vol. 386, 1997, pp. 474-477. doi:10.1038/386474a0 